\title{
A CASE OF BILATERAL PARASTERNAL DIAPHRAGMATIC HERNIA
}

BY

\author{
R. WARWICK BROWN \\ From St. Bartholomew's Hospital Medical College, London
}

(RECEIVED FOR PUBLICATION MARCH 21, 1952)

Amid the several recognized types of diaphragmatic hernia the incidence of the parasternal variety is sufficiently low to render an example of its bilateral occurrence worthy of record. This extremely rare condition was encountered during the recent routine examination of a dissectingroom cadaver, a female, aged 83, in the Department of Anatomy of St. Bartholomew's Hospital Medical College.

\section{Clinical History}

In life the patient had displayed no symptoms directly referable to the unsuspected diaphragmatic hernia. She had suffered from chronic bronchitis with alleged emphysema, but had complained of no digestive or other alimentary symptoms. The patient's obesity rendered it unlikely that any clear physical signs indicative of the condition could have been detected on clinical examination, and at no time of life did any suspicion arise of gross visceral displacement. She died of myocarditis and advanced senile change.

\section{THE HERNiAE}

General Account.-The abdomen being opened, a gross alteration of visceral topography was immediately apparent, a considerable portion of the bowel being found to occupy the right side of the chest. On this side a loop of gut, consisting of terminal ileum, caecum, and proximal colon, together with a portion of the great omentum, ascended anterior to the liver and entered the thorax through a parasternal diaphragmatic defect. On the left an additional small colonic loop ascended anterior to the left lobe of the liver and entered the thorax through a second parasternal diaphragmatic defect. In each instance the herniated organs lay within a complete peritoneal hernial sac. No other visceral displacement was present: the stomach and spleen were normally situated and the retroperitoneal organs were undisturbed.

The Diaphragmatic Defects.-The two diaphragmatic defects (Fig. 1) were oval, with long axes disposed transversely, and were bilaterally situated immediately behind the seventh costal cartilage. being separated by the xiphisternal portion of the diaphragm. The right and larger defect measured
$7 \mathrm{~cm}$. by $4.5 \mathrm{~cm}$. and the left defect $3.5 \mathrm{~cm}$. by $2 \mathrm{cco}$. Their orifices were bounded anteriorly by the seven $\mathbb{R}$ costal cartilage, laterally by the costal fibres of the diaphragm, and posteriorly by the anterior leaf of the central diaphragmatic tendon. They were sepa융 ated by three distinct muscular slips attaching the central tendon to the back of the xiphisternum, an these were disposed with a convexity to the left $\frac{}{a s}$ the larger dextral defect extended across the midline behind the xiphisternum. The superior epigastrif vessels entered the rectus sheath through the anterolateral margin of each defect.

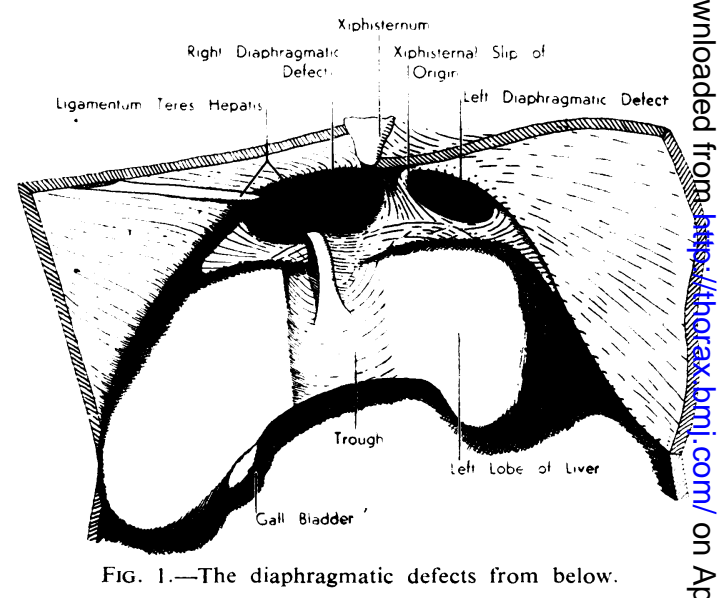

The Hernial Sacs.-Each hernial sac (Fig. 2) was continuous with the peritoneum clothing the did phragm and anterior abdominal wall. The sacs weres complete and were discrete from the pleural and pere cardial cavities. The large dextral sac invaginated the diaphragmatic pleura and extended as high as the second costal cartilage, the fundus being distant soms $15 \mathrm{~cm}$. from the neck. The smaller sinistral saf extended upwards and to the left for some 6 crm into the extrapericardial fat of the anterior mediastinum and did not contact the pleura.

The ligamentum teres hepatis and the falciforr ligament were incorporated in the wall of the large? sac. These structures entered the sac on its later $\$$ side, curving first upwards and backwards, and the medially and downwards to escape over the posteriof 


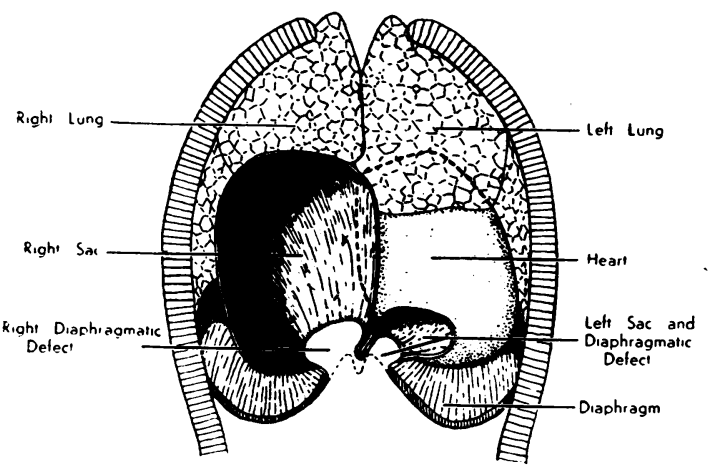

Fig. $2 a$

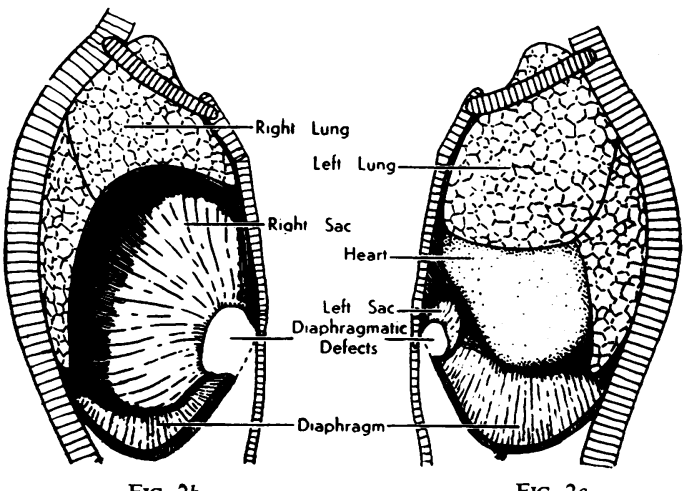

Fig. $2 b$

Fig. $2 c$

FIG. 2.-The hernial sacs and thoracic viscera: (a) anterior aspect; (b) right lateral aspect; (c) left lateral aspect.

margin of the diaphragmatic defect to reach a trough on the anterior aspect of the liver.

Sac Contents.- The large dextral sac (Fig. 3) contained some $50 \mathrm{~cm}$. of the terminal ileum, the entire caecum and appendix, some $60 \mathrm{~cm}$. of the proximal colon, and part of the great omentum. The small sinistral sac contained a short loop of colon continuous with the distal end of the colon in the other sac. There were a few peritoneal adhesions within the large sac but no evidence of obstruction: reduction of the sac contents into the abdomen was impossible without first enlarging its neck by cutting through the costal margin.

The entire colon had retained its primitive mesentery and the vessels therein were of normal origin and colonic distribution. A splenic flexure was lacking, the colon, on leaving the smaller sac, passing straight down to the left iliac fossa to become continuous with the pelvic colon.

Effects of the Herniae on Other Viscera.-The liver (Fig. 1) showed considerable widening of the ligamentum teres fissure, which took the form of a vertical trough some $4 \mathrm{~cm}$. wide in its anterior margin between the right and left hepatic lobes. The trough was occupied by the bowel entering and leaving the large sac, the ileum lying to the right of the colon in this situation. The ligamentum teres passed forwards and downwards over the posterior margin of the diaphragmatic defect and was attached to the liver in the floor of the trough.

The right lung (Fig. 2b) was small and deformed by the large dextral hernia. The interlobar fissures were markedly incomplete, but dissection of the bronchial tree revealed a normal pattern of bronchopulmonary segments. The segmental bronchi, particularly those of the middle lobe, showed a distortion commensurate with the general compression of the lung. The lower lobe extended caudally behind the hernia into a costo-diaphragmatic recess of normal extent. The pulmonary vessels were of normal disposition.

The left lung (Fig. 2c) was larger than the right, although it was somewhat compressed by the cardiac displacement. It consisted of the customary two lobes with a complete oblique interlobar fissure. The anatomy of the bronchial tree and the pulmonary vessels showed no abnormality.

The heart and pericardium (Fig. 2a) were displaced to the left so that the right cardiac border lay in the median plane and the cardiac apex some $3 \mathrm{~cm}$. lateral to the left mid-clavicular line. The lower part of its right ventricular region was displaced somewhat backwards by the small sinistral hernia. The outer wall of the right atrium was concave and the inferior vena cava entered the atrium from the right infero-lateral aspect.

\section{REPORTS IN THE LITERATURE}

A brief review of the literature reveals the rarity of the parasternal variety, which would appear to account for less than $3 \%$ of all diaphragmatic herniae. No previous report of its bilateral occurrence has been found.

Thoma (1882) describes four cases of diaphragmatic hernia of various types, one of which is

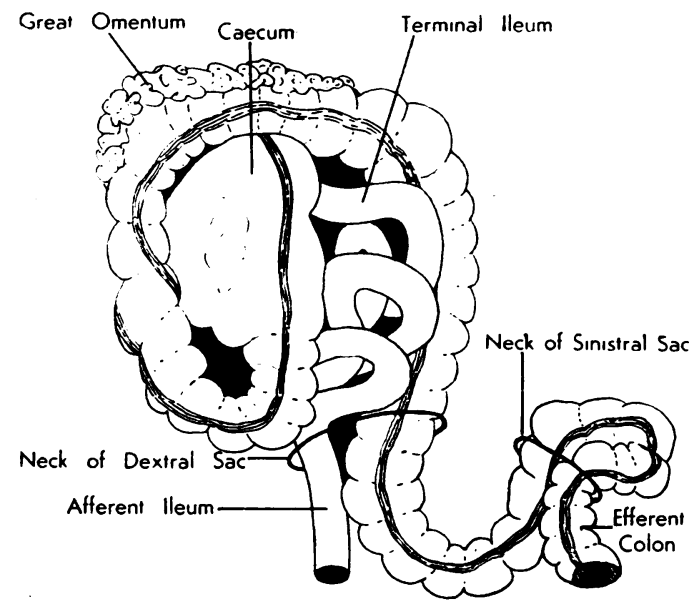

FIG. 3.-The contents of the hernial sacs. 
parasternal and closely resembles the dextral hernia of the present specimen, its sac containing the terminal ileum, caecum, and proximal colon. He reviews a total of 291 cases of diaphragmatic hernia, 26 of which he describes as " true herniae," i.e., possessing a peritoneal sac, and of these, eight are parasternal (six right-sided and two left-sided).

Keith (1910) reports on 34 museum specimens of diaphragmatic hernia, none of which is parasternal.

Kratzeisen (1921) describes a single example of left-sided parasternal hernia in an 83-year-old man. The peritoneal sac contained transverse colon and lay in the anterior mediastinum in front of the pericardium and thus closely resembled the condition encountered in the left side of the present subject.

Hume (1922) regards hernia through the foramen of Morgagni (space of Larrey) as a rare variety of diaphragmatic hernia, stating that only eight recorded cases were found in the literature, presumably those enumerated by Thoma.

Barrett and Wheaton (1934) refer to the rare class of diaphragmatic hernia through neural and vascular foramina and account parasternal hernia the least rare variety within this class, but say nothing further.

Dunhill (1935) reports 25 cases of diaphragmatic hernia, which include one case of parasternal hernia treated successfully by operation. In this patient, a man aged 29 , the operative findings were as follows :

"There was an hiatus 4 in. long behind the xiphisternum, apparently central in situation with a tendency to lie towards the right side. The peritoneal sac contained the termination of the ileum, the caecum, appendix, ascending and transverse colon. The sac and its contents were in the anterior mediastinum resting against the pericardium on the left side and displacing the right pleura and lung backwards and to the right. The neck of the sac had spread the costo-sternal fibres of the diaphragm widely."

These findings show a considerable similarity to the condition in the right side of the present subject.

Harrington (1942) reviews 304 examples of diaphragmatic hernia treated by operation at the Mayo Clinic: five of these (about 2\%) are described as "subcosto-sternal" (parasternal) in type. In one of these cases the hernial sac contained colon, caecum, appendix, terminal ileum, and great omentum, as in the present specimen. Of the others, one contained omentum and a loop of colon, while in the remainder the contents were omentum and fat.
Meyer (1950) reviews the subject of diaphrag matic hernia and describes "subcosto-sternal 'O hernia as the rarest type, giving, however, nof statistics.

\section{Discussion And Conclusions}

Parasternal hernia is, then, one of the rarest forms of diaphragmatic hernia and no previousu record of its bilateral occurrence has been dis covered. It is clear that parasternal hernia is quite compatible with a normal life-span, as in the present example and in those described bX Thoma and Kratzeisen, and, further, that it maßs be completely symptomless. When, howevero symptoms occur, the hernia is susceptible of radiological diagnosis and surgical cure (Dunhill, $1935 \mathrm{cs}$ Harrington, 1942).

Owing to its great rarity, little work has bee done to elucidate the aetiology. The diaphrag matic defect occurs between the costal and xiphisternal origins of the diaphragm at the poinf where the superior epigastric vessels escape from the thorax to enter the rectus sheath, i.e., througg the space of Larrey. This is also the site of the ven tral part of the embryonal pleuro-peritoneal canafo and it thus seems reasonable to compare par\% sternal hernia with the more commonly occurring lumbo-costal hernia (through the pleuro-peritonea hiatus of Bochdalek), which is acknowledged to bo due to defective closure of the dorsal part of the canal. In this latter site the pleuro-peritone membrane may"fail to close the canal completer with a resultant massive herniation of the abdo minal viscera incompatible with life (Dunhi夜, 1935). On the other hand, the canal may be closed by the pleuro-peritoneal membrane, bu subsequent failure of diaphragmatic muscle dev\& lopment may lead to herniation of abdomina viscera within a peritoneal sac, a condition conpatible with life. The parasternal herniae described to date have all possessed a complete peritone sac, and Dunhill correctly concludes therefore tha this hernia is not the result of a persistent embros onal pleuro-peritoneal hiatus. It appears (a poine Dunhill omits) that parasternal hernia may be paralleled by the second type of lumbo-cost hernia and be due to defective muscular reinforcement of the pleuro-peritoneal membrane. Tige recording of parasternal hernia in adult subjects only does not imply the absence of a primary congenital defect, as such defect may becones apparent only in later life. It would seem that tre explanation of parasternal hernia lies primarily the presence of a congenital structural defect 
that portion of the diaphragm which results from the fusion of septum transversum and body-wall components.

\section{SUMMARY}

A case of bilateral parasternal diaphragmatic hernia in an elderly woman is described.

No previous report of a similar condition has been found in the literature.

Parasternal hernia is the rarest type of diaphragmatic hernia and probably accounts for less than $3 \%$ of all specimens.

The probable explanation of parasternal hernia is the presence of a congenital structural defect of that portion of the diaphragm which results from the fusion of septum transversum and bodywall components.

My thanks are due to Professor A. J. E. Cave for permission to publish this report, to Dr. C. E. H. Milner, of Reigate, Surrey, for the clinical history, and to Professor Cave and Dr. H. Butler for assistance in the preparation of this report.

REFERENCES

Barrett, N. R., and Wheaton, C. E. W. (1934). Brit. J. Surg., 21, 420

Dunhill, T. (1935). Ibid., 22, 475.

Harrington, S. W. (1942). Ann. Surg., 115, 705.

Hume, J. B. (1922). Brit. J. Surg., 10, 207.

Keith, A. (1910). Brit. med. J., 2, 1297.

Kratzeisen. E (1921). Virchows Arch. path. Anat., 232, 227.

Meyer, H. W. (1950). J. thorac. Surg., 20, 235.

Thoma, R. (1882). Virchows Arch. path. Anat., 88, 515. 